\title{
Bedside Tracheostomy: Our Experience in a Tertiary Care Hospital
}

\author{
Vikas Sinha $^{1}\left(\mathbb{D} \cdot\right.$ Sushil G. Jha $^{1} \cdot$ Samanth Talagauara Umesh $^{1} \cdot$ Nirav P. Chaudhari $^{1}$ • \\ Bhagirathsinh D. Parmar ${ }^{1} \cdot$ Rashmin S. Patel $^{1}$
}

Received: 25 June 2020/ Accepted: 13 July 2020/Published online: 20 July 2020

(C) Association of Otolaryngologists of India 2020

\begin{abstract}
Tracheostomy is the creation of a stoma at the surface of skin, which leads into trachea. In the critically ill patients, it is one of the most frequently done procedure especially in intensive care unit (ICU) for those requiring prolonged mechanical ventilation. About $24 \%$ of all patients in ICU need tracheostomy (Esteban et al. in Am J Respir Crit Care Med 161:1450-1458, 2000). Historically it had a high complication rate and so many authors suggested that it should be done only in operating room (Dayal and Masri in Laryngoscope 96:5862, 1986). A standardized procedure to reduce complications was described by Jackson (Laryngoscope 19:285-290, 1909). The aim of the study is to observe and analyze the outcome of bedside open tracheostomy, in relation to its safety, complications and simplicity. Study consists of 200 patients who underwent bedside tracheostomies in a tertiary care center from 2014 to 2017 in medical/surgical/paediatric ICU's. All the
\end{abstract}

Vikas Sinha

vikassinha1958@gmail.com

Sushil G. Jha

sushilkgjhatanmay@gmail.com

Samanth Talagauara Umesh

moresam.12@gmail.com

Nirav P. Chaudhari

nirav1331993@gmail.com

Bhagirathsinh D. Parmar

drbhagirath89@gmail.com

Rashmin S. Patel

patelrashminsh12@gmail.com

1 Department of Otorhinolaryngology, Head and Neck Surgery, Sir T Hospital and Government Medical College, B303, Himalaya, Skyz Flat, Behind Himalaya Mall, Bhavnagar, Gujarat 364001, India procedures followed a standard protocol. In all the surgeries, two E.N.T. surgeons were scrubbed and did the procedure, assisted by two ICU nurses. One anesthetist who administered sedation and monitored the patient. If coagulation disturbances were present in elective case then they were corrected prior to the procedure. We all want the latest, safest, simplest and cheapest available technique in medical practice. Bedside tracheostomy is one such procedure. It is better than tracheostomy in operating room for patients who need prolonged mechanical ventilation in ICU as it eliminates the need of patient transport to OR and its associated complications and also minimizing cost. Training programs need to be provided to the assisting staff for better procedural outcome.

Keywords Tertiary care - Tracheostomy - Stenosis · ICU $\cdot$ Stoma

\section{Introduction}

Tracheostomy is the creation of a stoma at the surface of skin, which leads into trachea. In the critically ill patients, it is one of the most frequently done procedure especially in Intensive Care Unit (ICU) for those requiring prolonged mechanical ventilation. About $24 \%$ of all patients in ICU need tracheostomy [1]. Historically it had a high complication rate and so many authors suggested that it should be done only in operating room [2]. A standardized procedure to reduce complications was described by Jackson [3]. In 1950, Sheldon [4] introduced a newer method of Percutaneous tracheostomy which had many techniques over the years [5]. The technique described by Ciaglia [6] is being currently used most often. Of late tracheostomies done bedside in ICU's are proven to be safe [7]. 
Tracheostomy allows for patient mobilization, helps in feeding, physical and occupational therapy and decreases the need of sedation when compared to keeping patient intubated for prolonged duration. Bedside tracheostomy is convenient and offers scheduling flexibility. It can be done without any coordination with operating room in normal business hours. Also bedside tracheostomy obviates the transfer of critically ill patients to operating room [8]. In a study Massick et al. [9] found excellent results in bedside tracheostomy and suggested that it reduces the patient charge and provides a more secure airway. The aim of this study is to highlight the advantages and disadvantages encountered during bedside tracheostomy.

\section{Materials and Methods}

Study consists of 200 patients who underwent bedside tracheostomies in a tertiary care center from 2014 to 2017 in Medical/Surgical/Paediatric ICU's. All the procedures followed a standard protocol. In all the surgeries, two E.N.T. surgeons were scrubbed and did the procedure, assisted by 2 ICU nurses. 1 Anaesthetist who administered sedation and monitored the patient. If coagulation disturbances were present in elective case then they were corrected prior to the procedure.

\section{Preparation of the Patient}

Before starting the procedure, ventilator was changed to a set rate of at least 10 and Fio2 increased to $100 \%$. Intravenous sedation by Midazolam and Fentanil was given by anaesthetist on surgeon's request in un-cooperative and anxious patients. Blood pressure, cardiac rhythm and arterial haemoglobin saturation were monitor by the anaesthetist continuously throughout the procedure. The neck was hyperextended with the help of a shoulder bag and head supported with a head ring (removed in patients needing more extension). Aseptic surgical field was achieved by painting with betadine solution first followed by spirit. Drapes were applied. Local anaesthetic (lignocaine with adrenaline 1:200,000) agent was injected into the neck soft tissues around the incision site to cause anaesthesia and to reduce bleeding.

\section{Bedside Surgical Tracheostomy Technique}

A vertical $2 \mathrm{~cm}$ skin incision placed in the midline just above the suprasternal notch. Skin and subcutaneous tissues divided up to the depth of strap muscles. Strap muscles are separated vertically by blunt dissection. It is necessary at each step to palpate for the trachea and keep the surgical site clear of blood by repeated mopping.
Subsequently thyroid isthmus is seen which is retracted superiorly. Then the tracheal rings can be palpated. Trachea can be confirmed by presence of bubbles after introducing a half empty syringe in at and withdrawing it. Next the tracheal incision is generally kept through the second to fourth tracheal rings. This is followed by dilating the tracheal opening with a tracheal dilator. After dilating the trachea, appropriate sized tracheostomy tube is introduced along with simultaneous withdrawing of endotracheal tube in intubated patients. Following this tracheostomy tube is held in its position either by silk stay sutures or tying it with gauze piece. Betadine gauze piece is kept below the tracheostomy tube to prevent skin necrosis below the tracheostomy tube. Correct position of tube is confirmed by checking for movement of gauze piece placed in front of tube, maintaining of oxygen saturation, and lastly the presence of bilateral equal air entry. Chest X-ray was taken the next day to confirm the correct tube placement and to look for pneumothorax if any.

\section{Results}

The study group had 200 patients. It included 125 males, 55 females and 20 children ( $<12$ years). Maximum patients belonged to 31-50 years of age. Among the various departments in which bedside tracheostomy (both elective and emergency) needed to be done, majority patients were in ENT dept. $(39 \%, \mathrm{n}=78)$ followed by Medical ICU $(26.5 \%, \mathrm{n}=53)$, then Surgical ICU $(17 \%$, $\mathrm{n}=34$ ) and paediatric ICU and Burns ward. The most common cause for bedside tracheostomy was prolonged intubation (70\%, $\mathrm{n}=140)$; this included Road Traffic Accident with head injury, Cardio Vascular stroke, Accidental fall down, Organophosphate poisoning, Guillan Barre Syndrome, Chronic Obstructive Pulmonary Disease, Acute Respiratory Distress Syndrome. Other causes were Cancer patients $(22 \%, \mathrm{n}=44)$ of larynx, buccal mucosa, tongue which sometimes needed a prophylactic pre-operative tracheostomy. Few patients were of Burns. Paediatric causes $(8 \%, \mathrm{n}=16)$ were Tetanus, Diphtheria. The mean operative time was 19 min (range 8-40 min).

We faced many major and minor complications during tracheostomy. One of the most common was minor bleeding following either skin incision or after separating the strap muscles due to tissue disruption of the neck. This was managed with either tying the bleeder or using monopolar cautery (Table 1).

Stromal infection within a month was very frequent and hence associated with significant morbidity. In this study the incidence of stromal infection was in $5 \%$ of patients, which is less than that in literature [10]. Low infection rate in our study was due to careful separation and not cutting 
Table 1 Complications seen in bedside tracheostomy

\begin{tabular}{lcc}
\hline Complications & No. of patients & Time \\
\hline Hemorrhage & 12 & Within 72 h of surgery \\
Surgical emphysema & 5 & Within 6 h of surgery \\
Infections at stoma & 10 & Within 1 month \\
Cardiac arrest & 3 & During surgery \\
Pneumothorax & 4 & Within 1 day \\
Laryngotracheal stenosis & 2 & After 1 month \\
Tracheo-oesophageal fistula & 0 &
\end{tabular}

of neck tissues. This happens due to unsterile instruments or procedure. Once diagnosed we send the pus for culture and sensitivity followed by appropriate antibiotic therapy and regular sterile dressing. Few patients had surgical emphysema. This is due to a large opening in the trachea more than the diameter of the tracheostomy tube or due to a large skin incision. Its management is by manual removal of subcutaneous emphysema by pressure and reducing the length of skin incision by suturing.

Cardiac arrest was seen in three patients. These were patients with dyspnea for a long duration. There respiratory drive was $\mathrm{CO} 2$ dependent. Therefore, as soon as the tracheal incision was placed, $\mathrm{CO} 2$ escaped and patient went into cardiac arrest. Two of the three patients could be revived by CPCR, but one succumbed to death. The incidence of post cannulation stenosis was also low [11] due to avoidance of tracheal ring resection. This is in line with larger studies, which have reviewed complications, including a meta-analysis of outcomes for surgical procedures [7, 9].

\section{Discussion}

Simpson et al. [12] stated that out of fear of transporting ill patients tracheostomies were less frequently used in the past. So it was preferred to do tracheostomies in Operating Room (OR) instead of bedside due to better lighting, sterile and adequate instruments and support facilities [13]. Problems encountered in this are hazard of moving critically ill patients, [14] the cost and inconvenient OR schedules. But in a study by Henrich it is shown that transportation is not a critical issue [12] Also in many hospitals The number of ICU beds are limited [2, 13, 16] and patients with ventilators are kept in regular wards $[2,14,15]$. In addition, there is delay in performing tracheostomy in Operating theatre due to less number of OR and more elective and emergency procedures. This causes an overall lengthening the hospital stay of patients, delaying the weaning process [2, 15-18] and patient's morbidity is increased [2, 17, 19].

Doing tracheostomy bedside is less time consuming. It is safe in ICU patients. Study by Massick et al. concluded that bedside tracheostomy provides a secure airway at markedly reduced patient charges [9]. But there are a few inherent difficulties performing a bedside tracheostomy like Big sized ICU beds; few having water beds and hence not a firm surface to do tracheostomy, causing discomfort to the operating surgeon, inadequately trained staff for assistance, inadequate lighting, unsterile and insufficient instruments.

In a study by Bowen et al. [18] $2 \%(n=139)$ and in a study by Gysin et al. [19] the rate is $95 \%(\mathrm{n}=35)$, in our study it is $18 \%(n=200)$ which is acceptable. In a study by Upadhyay et al. [20] major bleeding occurred in $1 \%$ ( $n=470)$ of patients, whereas in our study there were no patients with major bleeding. Terra et al. [21] had $0.15 \%$ $(n=552)$ of infections at stoma site, but we have had $5 \%$ $(n=200)$ infections at stoma site.

Based on our study there are two crucial principles for best results in bedside tracheostomy. Firstly, the team should be well versed with tracheostomy. Many feel that tracheostomy is a simple procedure, but overconfidence and inexperience may lead to disastrous consequences. Secondly, there should be a standardized procedure and team cooperation between operating surgeon, anaesthetist and staff nurse. Adequate lighting and material should be there and everyone involved must be familiar with the steps of the surgical procedure.

\section{Conclusion}

We all want the latest, safest, simplest and cheapest available technique in medical practice. Bedside tracheostomy is one such procedure. It is better than tracheostomy in operating room for patients who need prolonged mechanical ventilation in ICU as it eliminates the need of patient transport to OR and its associated complications and also minimizing cost. Training programs 
need to be provided to the assisting staff for better procedural outcome.

\section{References}

1. Esteban A, Anzueto A, Alia I et al (2000) How is mechanical ventilation employed in the intensive care unit? An international utilization review. Am J Respir Crit Care Med 161:1450-1458 (Cross ref view record in Scopus citing articles (436))

2. Dayal VS, Masri W (1986) Tracheostomy in intensive care setting. Laryngoscope 96:5862

3. Jackson C (1909) Tracheostomy. Laryngoscope 19:285-290

4. Sheldon C, Pudenz R, Freshwater D (1955) A new method for tracheostomy. J Neurosurg 12:428-431

5. Powell DM, Price PD, Forrest LA (1998) Review of percutaneous tracheostomy. Laryngoscope 108:170-177

6. Bikhaze N (2001) Percutaneous tracheostomy: has its time arrived? Arch Otolaryngol Head Neck Surg 127:221-223

7. Dulguerov P, Gysin C, Perneger T et al (1999) Percutaneous or surgical tracheostomy: a meta-analysis. Crit Care Med 27:1617-1625

8. Henrich D, Blythe W, Weissler M et al (1997) Tracheotomy and the intensive care unit patient. Laryngoscope 107:844-847

9. Massick DD, Yao S, Powell DM, Griesen D, Hobgood T, Allen JN et al (2001) Bedside tracheostomy in the intensive care unit: a prospective randomized trial comparing open surgical tracheostomy with endoscopically guided percutaneous dilational tracheostomy. Laryngoscope 111:494-500

10. Muttini S, Melloni G, Gemma M et al (1999) A prospective, randomised evaluation of early and late complications after either percutaneous or surgical tracheostomies. Min Anestesiol 65:521527

11. Walz MK (2002) Tracheostomy. Indications, methods, risks. Anaesthesist 51:123-133
12. Simpson T, Day C, Jewkes C et al (1999) The impact of percutaneous tracheostomy on intensive care unit practice and training. Anaesthesia 54:186-189

13. Upadhyay A, Maurer J, Turner J, Tiszenkel H, Rosengart $\mathrm{T}$ (1996) Elective bedside tracheostomy in the intensive care unit. J Am Coll Surg 183:515

14. Wease GL, Frikker M, Villalba M, Glover J (1996) Bedside tracheostomy in the intensive care unit. Arch Surg 131:5525

15. Hersch M, Sonnenblick M, Karlic A, Einav S, Sprung CL, Izbicki G (2007) Mechanical ventilation of patients hospitalized in medical wards vs. the intensive care UNITAN observational, comparative study. J Crit Care 22(1):13-17

16. Cox CE, Carson SS (2012) Medical and economic implications of prolonged mechanical ventilation and expedited postacute care. Sem Respir Crit Care Med 33(4):357-361

17. Hersch M, Izbicki G, Dahan D, Breuer GS, Nesher G, Einav S (2012) Predictors of mortality of mechanically ventilated patients in internal medicine wards. J Crit Care 27(6):694-701

18. Bowen CP, Whitney LR, Truwit JD et al (2001) Comparison of safety and cost of percutaneous versus surgical tracheostomy. Am Surg 67:54-60 (View record in Scopus citing articles (60))

19. Gysin C, Dulguerov P, Guyot JP et al (1999) Percutaneous versus surgical tracheostomy, a double-blind randomized trial. Ann Surg 230:708-714 (Cross ref view record in Scopus citing articles (106))

20. Upadhyay A, Maurer J, Turner J et al (1996) Elective bedside tracheostomy in the intensive care unit. J Am Coll Surg 183:51-55 (View record in Scopus citing articles (42))

21. Terra RM, Fernandez A, Bammann RH et al (2007) Open bedside tracheostomy: routine procedure for patients under prolonged mechanical ventilation. Clinics 62:427-432 (Cross ref view record in Scopus citing articles (7))

Publisher's Note Springer Nature remains neutral with regard to jurisdictional claims in published maps and institutional affiliations. 